\title{
Dirac spinor in a nonstationary Gödel-type cosmological universe
}

\author{
Víctor M. Villalba \\ Centro de Física \\ Instituto Venezolano de Investigaciones Científicas, IVIC \\ Apdo 21827, Caracas 1020-A, Venezuela
}

\begin{abstract}
In the present article we solve, via separation of variables, the massless Dirac equation in a nonstationary rotating, causal Gödel-type cosmological universe, having a constant rotational speed in all the points of the space. We compute the frequency spectrum. We show that the spectrum of massless Dirac particles is discrete and unbounded.
\end{abstract}


During the last years there has been a growing interest in study of quantum effects associated with scalar and spinor particles in cosmological universes. In this direction a particular attention has been devoted to the analysis of homogeneous universes as well as of rotating space-times.

Obviously, in order to analyze quantum effects on curved backgrounds is necessary to carry out a careful study of the one-particle states, that is, a detailed investigation of the exact solution of the relativistic wave equations in curved space-times.

Despite the considerable effort dedicated to the search of exact solutions of relativistic wave equations in curved space-times, only a few examples are available in the literature where the solutions are expressed in closed form, being the background fields where this one is possible associated with diagonal metrics [1] or in the best ot the cases with the type D Petrov spaces[2]. A different family of universes, where scalar particles as well as Dirac particles have been analyzed, is the so-called Gödeltype space-times. The interest in this kind of models is related to the study of the possible effects of the rotation in the formulation of a quantum field theory on curved spaces. In this framework some exact solutions of the massless Dirac equation

$$
\gamma^{\alpha} \nabla_{\alpha} \Psi=0
$$

with the chirality condition

$$
\left(1-i \gamma_{5}\right) \Psi=0
$$

have been obtained. Regretfully, due to the structure of the system of equations to be decoupled and solved, no exact solutions in terms of special functions are available for the massive case. Among the space-times where (11) and (2) have been solved we have the Gödel universe [3]

$$
d s^{2}=-\left(d t+e^{a r} d \theta\right)^{2}+d r^{2}+\frac{1}{2}\left(e^{a r} d \theta\right)^{2}+d z^{2},
$$

also the universe associated with The Einstein-Maxwell equations in the presence of a perfect fluid and a sinusoidal electromagnetic field with vanishing rest charge density (4) 


$$
d s^{2}=-(d t+a x d y)^{2}+d x^{2}+d y^{2}+d z^{2}
$$

other metrics where the Klein-Gordon and the Weyl equation are also soluble in terms of hypergeometric functions are [5]

a) The Som-Raychaudhuri metric

$$
d s^{2}=-d t^{2}+d r^{2}+d z^{2}-2 r^{2} d \phi d t+r^{2}\left(1-r^{2}\right) d \phi^{2}
$$

b) The Hoenselaers-Vishveshwara metric

$$
d s^{2}-d t^{2}+d r^{2}+d z^{2}-\frac{1}{2}(c-1)(c-3) d \phi^{2}-2(c-1) d \phi d t
$$

c) The Rebouças metric

$$
d s^{2}=-d t^{2}+d r^{2}+d z^{2}+4 \cosh 2 r d \phi d t-\left(3 \cosh ^{2} 2 r+1\right) d \phi^{2}
$$

in all the above cases the scalar wave as well as the Dirac spinor present an oscillating behavior in the radial variable.

Recently [6] the massless Klein-Gordon equation.

$$
\nabla^{\alpha} \nabla_{\alpha} \Phi=0
$$

has been studied in a cosmological rotating model associated with the metric,

$$
d s^{2}=-d t^{2}+c^{2} t^{2}\left(d x^{2}+\lambda e^{2 m x} d y^{2}+d z^{2}\right)+2 c t e^{m x} d y d t
$$

where $\mathrm{c}, \lambda$, and $m$ are positive constants The rotational speed of the model given by eq. (9) is

$$
\omega=\frac{m}{2 c t \sqrt{\lambda+1}}
$$

Among the advantages of the metric (9) we have that the model is causal , i.e., there are not closed timelike lines. Exact solutions of eq. (8), and the corresponding frequency spectrum analysis for the metric (9) with $\lambda=0$, have been presented by 
Panov[7]. It is the purpose of the present article to obtain exact solutions of the massless Dirac equation in the background field given by eq. (9) (even for $\lambda \neq 0$ ) and to compute the corresponding frequency spectrum.

The covariant generalization of the massless Dirac equation in curved space-time is,

$$
\left[\gamma^{\mu}\left(\partial_{\mu}-\Gamma_{\mu}\right)\right] \Psi=0
$$

where the curved Dirac matrices satisfy the anticommutation relations

$$
\left\{\gamma^{\mu}, \gamma^{\nu}\right\}_{+}=2 g^{\mu \nu}
$$

and the spinor connection $\Gamma_{\lambda}$ is $[$ ]

$$
\Gamma_{\lambda}=\frac{1}{4} g_{\mu \alpha}\left(\partial_{\lambda} h_{\nu}{ }^{k} h_{k}^{\alpha}-\Gamma_{\nu \lambda}^{\alpha}\right) s^{\mu \nu} .
$$

In order to compute (13) we have to choose a particular tetrad associated with the line element given by (9). A suitable election is,

$$
\gamma^{0}=\tilde{\gamma}^{0}+\frac{1}{\sqrt{\lambda+1}} \tilde{\gamma}^{2}, \gamma^{1}=\frac{1}{c t} \tilde{\gamma}^{1}, \gamma^{2}=\frac{e^{-m x}}{c t \sqrt{\lambda+1}} \tilde{\gamma}^{2}, \tilde{\gamma}^{3}=\frac{1}{c t} \tilde{\gamma}^{3},
$$

after substituting (14) into (13), we have that the Dirac equation takes reads,

$$
\left.\left\{\tilde{\gamma}^{0}+\frac{1}{\sqrt{\lambda+1}} \tilde{\gamma}^{2}\right) \partial_{t}+\frac{1}{c t} \tilde{\gamma}^{1} \partial_{x}+\frac{e^{-m x}}{c t \sqrt{\lambda+1}} \tilde{\gamma}^{2} \partial_{y}+\frac{1}{c t} \tilde{\gamma}^{3} \partial_{z}\right\} \Phi=0,
$$

where we have introduced the spinor $\Phi$ given by the expression

$$
t e^{m x / 2} \Psi=\Phi
$$

After introducing the new time variable $\eta$ which is related to $t$ by the expression

$$
t=\exp (c \eta)
$$

we have that (15) takes the form

$$
\left\{-\omega \tilde{\gamma}^{0}-i \tilde{\gamma}^{1} \partial_{x}+\frac{1}{\sqrt{\lambda+1}}\left(\frac{k_{y}}{e^{m x}}-\omega\right) \tilde{\gamma}^{2}+k_{z} \tilde{\gamma}^{3}\right\} \Phi_{0}=0,
$$


where the spinor $\Phi_{0}$ is related to $\Phi$ as follows,

$$
\Phi=\Phi_{0} e^{i\left(k_{y} y+k_{z} z-\omega t\right)}
$$

the operator (18) can be written as a sum of two commuting operators $\hat{K}_{1}$ and $\hat{K}_{2}$ given by the expressions 9

$$
\begin{gathered}
\hat{K}_{1}=\left(-\omega \tilde{\gamma}^{0}+k_{z} \tilde{\gamma}^{3}\right) \tilde{\gamma}^{0} \tilde{\gamma}^{3}, \\
\hat{K}_{2}=\left(-i \tilde{\gamma}^{1} \partial_{x}+\frac{1}{\sqrt{\lambda+1}}\left(\frac{k_{y}}{e^{m x}}-\omega\right) \tilde{\gamma}^{2}\right) \tilde{\gamma}^{0} \tilde{\gamma}^{3},
\end{gathered}
$$

where $\hat{K}_{1}$ and $\hat{K}_{2}$ act on the spinor $\Xi$ as follows,

$$
\hat{K}_{1} \Xi=-\hat{K}_{2} \Xi=i \kappa \Xi, \quad \Xi=\tilde{\gamma}^{0} \tilde{\gamma}^{3} \Phi_{0}
$$

Choosing to work in the Jauch and Rohrlich Dirac matrices' representation,

$$
\tilde{\gamma}^{0}=\left(\begin{array}{cc}
-i & 0 \\
0 & i
\end{array}\right), \tilde{\gamma}^{i}=\left(\begin{array}{cc}
0 & \sigma^{i} \\
\sigma^{i} & 0
\end{array}\right), i=1,2,3
$$

we have that, taking into account (20) and (23), the spinor $\Xi$ takes the following block structure.

$$
\Xi=\left(\begin{array}{c}
\Xi_{1} \\
\Xi_{2}
\end{array}\right)=\left(\begin{array}{c}
\Xi_{1} \\
\frac{-i \omega}{k_{x}+\kappa} \sigma_{3} \Xi_{1}
\end{array}\right), \quad \Xi_{1}=\left(\begin{array}{c}
\alpha \\
\beta
\end{array}\right)
$$

where the constant of separation appearing in (22) satisfies the relation,

$$
\kappa^{2}=k_{z}^{2}-\omega^{2}
$$

after substituting (24) into $\hat{K}_{1} \Xi=i \kappa \Xi$ with $\hat{K}_{1}$ given by (21) we obtain the following system of coupled differential equations,

$$
\begin{aligned}
& {\left[\frac{d}{d x}+\frac{1}{\sqrt{\lambda+1}}\left(\frac{k_{y}}{e^{m x}}-\omega\right)\right] \beta-i \kappa \alpha=0,} \\
& {\left[\frac{d}{d x}-\frac{1}{\sqrt{\lambda+1}}\left(\frac{k_{y}}{e^{m x}}-\omega\right)\right] \alpha+i \kappa \beta=0,}
\end{aligned}
$$


then, the spinor solution $\Psi_{c}$ of the massless Dirac equation with the chirality condition (目) can be written as follows,

$$
\Psi_{c}=e^{-(c \eta+m x / 2)}\left(1+i \gamma_{5}\right)\left(\begin{array}{c}
-\frac{\omega}{k_{x}+\kappa} \Xi \\
i \sigma^{3} \Xi
\end{array}\right)
$$

the solution of (26), (27) can be expressed in terms of confluent hypergeometric functions $M(a, b, z)$,

$$
\begin{gathered}
\alpha=-i a_{0} \frac{k_{z}-\omega}{m \sqrt{\lambda+1}} e^{-k_{y} \eta / m \sqrt{\lambda+1}} \eta^{k_{z} / m \sqrt{\lambda+1}} M\left(-\frac{\omega+k_{z}}{m \sqrt{\lambda+1}}+1, \frac{2 k_{z}}{m \sqrt{\lambda+1}}+1, \frac{2 k_{y} \eta}{m \sqrt{\lambda+1}}\right) \\
\beta=a_{0} e^{-k_{y} \eta / m \sqrt{\lambda+1}} \eta^{k_{z} / m \sqrt{\lambda+1}} M\left(-\frac{\omega+k_{z}}{m \sqrt{\lambda+1}}, \frac{2 k_{z}}{m \sqrt{\lambda+1}}+1, \frac{2 k_{y} \eta}{m \sqrt{\lambda+1}}\right)
\end{gathered}
$$

in order to have regular solutions of $\alpha$ and $\beta$ for any value of $\eta$, we must impose the condition

$$
\omega+k_{z}=(n+1) m \sqrt{\lambda+1}
$$

which determines the frequency spectrum for massless Dirac particles in the background field (9). We have to notice that the frequency spectrum is discrete when $k_{z}=0$

A cosmological model closed related to that one described by (9) is .

$$
d s^{2}=R^{2}\left(-d \tau^{2}+d x^{2}+d z^{2}+2 e^{m x} d y d \tau\right)
$$

where $R=R(\tau)$ is an arbitrary differentiable positive function, and the constant $m$ is different from zero. In the present case there is a null coordinate, and also an expansion factor $R(\tau)$ is present. In order to study the massless Dirac equation in the metric (32) we are going to work in the following tetrad gauge,

$$
\gamma^{0}=\frac{1}{R}\left(\tilde{\gamma}^{0}+\tilde{\gamma}^{2}\right), \gamma^{1}=\frac{1}{R} \tilde{\gamma}^{1}, \gamma^{2}=\frac{e^{-m x}}{R} \tilde{\gamma}^{2}, \tilde{\gamma}^{3}=\frac{1}{R} \tilde{\gamma}^{3}
$$

After computing the spinor connections and substituting them into (11) we arrive at,

$$
\left\{-\omega \tilde{\gamma}^{0}-i \tilde{\gamma}^{1} \partial_{x}+\left(\frac{k_{y}}{e^{m x}}-\omega\right) \tilde{\gamma}^{2}+k_{z} \tilde{\gamma}^{3}\right\} \Phi_{1}=0
$$


where $\Phi_{1}$ is,

$$
\Phi_{1}=e^{\frac{m x}{2}} e^{\frac{3}{4} R^{2}} \Psi .
$$

Noticing that (18) reduces to (34) when we put $\lambda=0$ we have that in the present case the frequency spectrum takes the form

$$
\omega+k_{z}=(n+1) m
$$

\section{References}

[1] G. V. Shishkin and V. M. Villalba, J. Math. Phys. 33 (1991) 2093

[2] N. Kamran and R. G. McLenaghan, J. Math. Phys. 25 (1984) 1019

[3] L.O. Pimentel and A. Macías, Phys. Lett. A. 117 (1986) 325

[4] U. Percoco and V. M. Villalba, Can. J. Phys. 69 (1991) 665

[5] K. D. Krori, P. Borgohain, P. K. Kar and D. Das, J. Math. Phys 26 (1988) 1645

[6] V. F. Panov, Izv Vuzov, Fizika 1 (1990) 62

[7] V. F. Panov, Izv. Vuzov. Fizika. 2 (1991) 54

[8] D. Brill and J. A. Wheeler, Rev. Mod. Phys. 29 (1957) 465

[9] G. V. Shishkin and V. M. Villalba, J. Math. Phys. 33 (1992) 2093

[10] J.M. Jauch and F. Rohrlich, The Theory of Photons and Electrons (Springer, New York, 1976) 